\title{
LA INTERACCIÓN ENTRE LA POLÍTICA MACROPRUDENCIAL Y LA POLÍTICA MONETARIA
}

\author{
Este artículo repasa los objetivos y posibles puntos de interacción entre la política \\ monetaria y la política macroprudencial. También revisa los desarrollos más recientes \\ respecto al debate sobre la idoneidad o no de coordinar ambas políticas. El trabajo \\ concluye con una visión de los principales retos que deben afrontar estas dos políticas \\ dadas las condiciones macroeconómicas actuales.
}

\section{The interaction between macroprudential and monetary policy}

This article reviews the objectives and possible points of interaction between monetary policy and macroprudential policy. It also goes through the recent debate on whether or not these policies should be coordinated. The paper concludes with an overview of the main challenges that these two policies face under current macroeconomic conditions.

Palabras clave: estabilidad de precios, estabilidad financiera, coordinación de políticas.

Keywords: price stability, financial stability, policy coordination.

JEL: E32, E44, E52, E58, G28.

\section{Introducción}

La crisis financiera global volvió a poner de manifiesto la relevancia de las variables financieras en los procesos de expansión y recesión económica. Tras un período de relativa calma conocido como la Gran Moderación ${ }^{1}$, las principales economías avanzadas se vieron sumergidas en una fuerte recesión. La gestión de la política económica, principalmente la política monetaria no consiguió aliviar la dureza de la caída ni la duración de la misma.

\footnotetext{
* Universidad Autónoma de Madrid.

Agradezco los comentarios de un evaluador anónimo, así como de Ángel Estrada sobre versiones anteriores que sin duda han ayudado a mejorar considerablemente este trabajo. Todos los errores que pueda haber son de mi responsabilidad.

Contacto: beatriz.deblas@uam.es

Versión de enero de 2021.

https://doi.org/10.32796/ice.2021.918.7159
}

De esta forma, la crisis financiera global volvió a traer a escena la importancia de las variables financieras que hasta entonces no formaban parte del enfoque principal de la política monetaria. Además, puso de manifiesto que la política monetaria tal y como la conocíamos no está diseñada para frenar burbujas en los precios de los activos o desequilibrios financieros en épocas de estabilidad de precios. Es por ello que el interés giró hacia otras políticas como la macroprudencial para atender

\footnotetext{
1 Se conoce como Gran Moderación al período que va desde mitad de los años ochenta hasta 2007 caracterizado por una menor volatilidad en los principales agregados macroeconómicos, sobre todo en producto e inflación. Entre los factores explicativos de esta menor volatilidad destaca el fuerte papel estabilizador de la política monetaria (Stock \& Watson, 2002; Clarida, Galí \& Gertler, 2000; de Blas, 2009; entre muchos otros). Durante este período, el objetivo mayoritario entre los bancos centrales se caracterizó por conseguir la estabilidad de precios, a través de metas claras de inflación (inflation targeting) y su principal instrumento, los tipos de interés a corto plazo.
} 
a las preocupaciones sobre la inestabilidad financiera (Constâncio, 2017).

La inestabilidad financiera de finales de la primera década de los años 2000 se tradujo en inestabilidad económica, planteando la necesidad de entender los ciclos financieros ${ }^{2}$ y su relación con los ciclos económicos reales. Gran parte de la literatura se ha centrado en caracterizar e identificar las variables clave en los ciclos financieros, así como su relación con los ciclos económicos.

Los ciclos financieros se suelen medir por fluctuaciones en el crédito, el precio de la vivienda y de los activos financieros. La evidencia muestra que estas variables financieras tienen cierta relevancia a la hora de explicar las fluctuaciones reales a nivel global (Dées, 2016). Jordà et al. (2017) analizan la interacción entre ciclos financieros y reales utilizando una base de datos macrofinancieros para 17 economías avanzadas durante los últimos 150 años. Sus resultados muestran que, tras la Segunda Guerra Mundial, los ciclos económicos reales y los ciclos financieros están más fuertemente sincronizados. Los ciclos de crédito son ligeramente más largos que los reales. Asimismo, ambos presentan una clara asimetría entre expansiones y recesiones, siendo los ciclos financieros más «violentos» que los reales. Además, cuando las fuentes de fluctuaciones de varias variables financieras coinciden temporalmente, los efectos se amplifican, en particular entre el crédito y los precios de la vivienda (Claessens, Kose \& Terrones, 2011).

Desde el punto de vista de política económica, si la política monetaria es parte de esta relación entre ciclos

\footnotetext{
2 Los ciclos financieros se entienden como fluctuaciones económicas que o bien se originan en o se ven amplificadas por el sistema financiero. Estos ciclos se caracterizan por ser más largos y volátiles que los ciclos económicos -véase Borio (2012) para un análisis detallado sobre los ciclos financieros y sus características-. Al igual que aquellos, los ciclos financieros se caracterizan por períodos de repunte (upturn) y otros de caídas o recesión (downturn). Los períodos de expansión se caracterizan por gran abundancia de crédito, precios de los activos altos (en ocasiones burbujas tanto en el mercado inmobiliario como en el mercado de acciones) y un aumento del riesgo asumido por los agentes en los mercados financieros (BCE, 2017). Estos períodos de boom suelen culminar en crisis financieras que se traducen en crisis económicas reales, es decir, las fluctuaciones financieras generan volatilidad en la economía real.
}

económicos y financieros, es importante entender cómo interactúa con la política prudencial o la política fiscal. Por ejemplo, fuertes cambios en el ritmo de crecimiento del crédito que se traduzcan en fluctuaciones en el PIB o la inflación pueden desencadenar reacciones por parte de la política monetaria. En tanto en cuanto esta reacción vaya en el mismo sentido que la de política macroprudencial (porque sus objetivos estén alineados), ambas se reforzarán mutuamente. Pero también pueden contrarrestarse una a la otra, reduciendo así la eficiencia de ambas políticas. Existe cierto consenso en la literatura de que las características de los ciclos financieros (tanto domésticos como globales) dependen de la estructura financiera del país, de la política monetaria y de la calidad de las instituciones (Estrada et al., 2017). Esta relación resultó clave para los efectos negativos de la crisis financiera global y en parte ha generado una mayor atención hacia los factores financieros como determinantes de fluctuaciones reales.

La principal conclusión es que es necesario contar con los efectos de los mercados financieros y desarrollar políticas enfocadas en ellos con el fin de evitar los efectos devastadores de futuras crisis financieras. Desde la crisis financiera global, el uso de políticas macroprudenciales en los países avanzados ha aumentado exponencialmente (Cerutti et al., 2017), con el fin de reducir la volatilidad del ciclo crediticio ${ }^{3}$.

Desde el punto de vista académico, el diseño de las nuevas políticas macroprudenciales ha inundado gran parte de los avances en materia de investigación macroeconómica de los últimos años. El objetivo es entender los efectos macroeconómicos de diseños alternativos de políticas macroprudenciales y sus efectos en la economía, cuando hay otras políticas como la

\footnotetext{
3 Es importante mencionar que el uso de políticas macroprudenciales estaba más generalizado en los países emergentes con anterioridad a la crisis financiera global. Estas economías sufrieron fuertes recesiones a finales de los años noventa, tras procesos de fuerte liberalización financiera. En estos países, las políticas macroprudenciales están activas y tienen como principal objetivo el proteger al sector financiero de fuertes fluctuaciones en los flujos de capital (véase Galati \& Moessner, 2013 y las referencias ahí citadas).
} 
fiscal y la monetaria también actuando. Sin embargo, aún no está claro el diseño óptimo de las mismas, entre otras razones, por las interacciones y efectos colaterales que dichas políticas tienen entre sí.

Hasta la crisis financiera global, el consenso era que los bancos centrales debían centrarse únicamente en un objetivo de inflación y solo tener en cuenta los desarrollos financieros en tanto en cuanto estos afectaran a la inflación. Este punto de vista cambió radicalmente tras la crisis (Constâncio, 2017; Freixas et al., 2015; Svensson, 2018). Se abrió entonces el debate de si la política monetaria debería incorporar entre sus competencias la de estabilidad financiera (Constâncio, 2017).

Este debate cobra especial relevancia en el seno de una unión monetaria como en la que nos encontramos. La eurozona ha optado por un diseño en el que la política monetaria se encuentra centralizada en el Banco Central Europeo dejando un mayor grado de descentralización respecto a la política macroprudencial. Todo ello, en medio de un proceso de avances hacia una unión bancaria que aún está sin completar. Comentaremos las implicaciones de este diseño más adelante, así como otras posibles alternativas, sus ventajas e inconvenientes.

Asimismo, la actual crisis del COVID-19 supone una fuerte caída de la actividad económica global que afecta a las posiciones deudoras de los agentes y, por consiguiente, a las hojas de balance de las entidades financieras. Con el fin de evitar el colapso del sistema bancario, los gobiernos han puesto en marcha importantes medidas prudenciales en combinación con las extraordinarias medidas monetarias y fiscales iniciadas en marzo de 2020. Aún está por ver la eficacia de tales medidas, pero sin duda nos proporciona un escenario perfecto para el estudio de las interacciones entre políticas ${ }^{4}$.

\footnotetext{
4 El Banco de España dedica su Informe de Estabilidad Financiera de primavera de 2020 a las características y consecuencias de esta crisis. Tras identificar los riesgos macrofinancieros que ha supuesto el COVID-19, el informe detalla la situación del sistema financiero español y evalúa su capacidad de respuesta, así como las políticas llevadas a cabo tanto micro como macroprudenciales. El objetivo fundamental de las medidas macroprudenciales llevadas a cabo era «mitigar el impacto de disrupciones financieras sistémicas, como la generada por el COVID-19, sobre la
}

En este artículo repasaremos los objetivos y posibles puntos de interacción entre la política monetaria y la política macroprudencial (apartado 2). También revisaremos los desarrollos más recientes al respecto y el debate sobre la idoneidad o no de coordinar ambas políticas (apartados 3 y 4). El objetivo es entender los posibles solapamientos y efectos colaterales de cada una de las políticas que llevan a plantearnos la necesidad y la idoneidad o no de la coordinación de ambas políticas, y su diseño institucional (apartado 5). Concluiremos con una visión de los principales retos que deben afrontar estas dos políticas dadas las condiciones macroeconómicas actuales y las posibles vías de avance.

\section{Objetivos de la política monetaria y de la política macroprudencial}

Antes de analizar las interacciones entre la política monetaria y la macroprudencial, es conveniente detallar los objetivos que gobiernan cada una de ellas. En este sentido, nos centraremos en los objetivos primarios para después pasar a detallar los efectos indirectos o colaterales que pueden en principio plantear posibles interacciones y conflictos.

\section{Objetivos primarios de política}

Los objetivos de la política monetaria difieren de país a país. En la mayoría de las economías industrializadas, los bancos centrales tienen un objetivo prioritario de estabilidad de precios $^{5}$. En algunos países (por ejemplo, en EE UU) este fin es dual y se complementa con un objetivo de crecimiento sostenido o desempleo en niveles bajos. En Europa, el Banco Central Europeo tiene un objetivo estricto de inflación.

economía real» garantizando el flujo de liquidez en el sistema, así como unas condiciones de acceso al crédito a hogares y empresas holgadas (Aguilar et al., 2020).

5 Véase Svensson (2011) para un análisis de la corriente de inflation targeting que ha dominado la gestión de la política monetaria en países industrializados desde principios de los años noventa. 
Dentro del arsenal de instrumentos que tienen los bancos centrales para alcanzar sus metas, el principal en tiempos normales es el tipo de interés a corto plazo ${ }^{6}$. Nos centraremos en este instrumento ya que afecta directamente al campo de actuación de la política macroprudencial. El banco central utiliza variaciones en el tipo de interés y la oferta monetaria para afectar finalmente a las variables objetivo a través de un mecanismo de transmisión monetario. Este mecanismo funciona a través de varios canales (tipos de interés, tipo de cambio, precio de los activos), siendo el sector financiero uno de los principales agentes, si no el más importante, en la transmisión de estas decisiones monetarias a la economía real. Por ello, desde el punto de vista de la política monetaria es crucial disponer de estabilidad financiera y de un sistema financiero que funcione bien con el fin de alcanzar su objetivo prioritario a través de sus instrumentos de política.

En tiempos de tipos de interés excesivamente bajos o con políticas monetarias no convencionales, como los que hay actualmente en vigor, el tipo de interés deja de ser un instrumento eficaz para la consecución de los objetivos de la política monetaria. En este caso, el uso de políticas monetarias no convencionales ofrece instrumentos cuyos efectos también repercuten en la estabilidad financiera o que incluso se asemejan a políticas macroprudenciales. Un ejemplo serían las facilidades crediticias dirigidas (como los TLTROs en la zona euro) que ofrecen financiación asequible a los bancos con el fin de estimular los préstamos a la economía real. Estas medidas no convencionales buscan proveer de más liquidez a determinados segmentos del mercado, además de reducir los tipos de interés y mejorar las condiciones de acceso al crédito de empresas e instituciones financieras. Sin embargo, dichas medidas no convencionales no están exentas de costes ya que pueden

\footnotetext{
6 Al controlar los tipos de interés a corto plazo, el mecanismo de transmisión en tiempos normales funciona alterando los tipos a largo plazo, así como tipos de cambio y precios de otros activos.

Svensson (2018) analiza en detalle cómo la política monetaria dispone de distintos instrumentos en tiempos normales y de crisis.
}

generar distorsiones en los mercados financieros, en la curva de rendimientos, además de potenciar la posición dominante del banco central en determinados mercados, como el de deuda soberana (Beirne et al., 2011). Por otro lado, también pueden interferir en los objetivos de estabilidad financiera al propiciar la excesiva toma de riesgo y posiciones de apalancamiento.

Respecto a la política macroprudencial, su principal objetivo es la estabilidad financiera (Nier \& Kang, 2016). La política macroprudencial «utiliza en primer lugar instrumentos prudenciales para mitigar el riesgo sistémico y de esta manera evitar la distorsión de los servicios financieros en la economía» (FSB-BIS-IMF, 2011). Este objetivo se puede cuantificar y medir por diferentes indicadores, los cuales determinan los distintos instrumentos para alcanzarlos. No pretendemos aquí hacer una descripción detallada de cada uno de los instrumentos de política macroprudencial ${ }^{7}$. Simplemente destacamos aquellos más relacionados con la política monetaria, entre ellos los requerimientos de reservas (oferta de crédito), techos en los préstamos demandados (demanda de crédito), así como en los créditos concedidos que incluyen los requerimientos de capital y ratios de préstamo a valor (loan-to-value ratios, LTV, por sus siglas en inglés) (Portes, 2014) ${ }^{8}$.

\section{Efectos colaterales}

Si bien la política monetaria y la macroprudencial no comparten, a priori, los mismos objetivos, sí que comparten mecanismos de transmisión. En este sentido, la coincidencia de variables entre ellas, tales como los tipos de interés o regulaciones del funcionamiento de los bancos (agentes clave en el mecanismo de transmisión monetaria), hace irremediable que las

\footnotetext{
7 Existen varias clasificaciones de los instrumentos de política macroprudencial en función de los objetivos que busquen, de las variables sobre las que se definen, sobre el sector de actividad al que van dirigidos, etc. Algunas referencias incluyen Blanchard et al. (2013) Banco de Inglaterra (2011) o Mencía y Saurina (2016), entre otros.

8 Una discusión detallada de los instrumentos primarios e intermedios se pueden encontrar en ESRB (2013).
} 
decisiones de política monetaria tengan efectos indirectos pudiendo potenciar o contrarrestar los objetivos de las políticas entre sí.

Algunos trabajos (e.g. Nier \& Kang, 2016; FMI, 2013) argumentan que los efectos de la política monetaria sobre la estabilidad financiera se plantean con incertidumbre y dependen, en parte, de la fase del ciclo financiero en que nos encontremos, así como del grado de apertura de la economía en cuestión. En particular, Nier y Kang (2016) ofrecen una clasificación bastante concisa de los distintos canales a través de los cuales la política monetaria puede afectar a la estabilidad del sistema financiero.

Analizaremos estos canales en detalle en el apartado 3, pero podemos avanzar que, por un lado, cambios en los tipos de interés pueden afectar al nivel de riesgo asumido por los agentes y a la demanda de crédito. Por otro lado, la política monetaria afecta también a las condiciones de los mercados financieros (oferta de crédito y su coste). Freixas et al. (2015) presentan evidencia de estas interacciones, y de cómo la política monetaria puede influir en el coste y oferta de crédito, así como en las posiciones de riesgo de los agentes. Los autores también indican que la evidencia sobre la efectividad de las políticas macroprudenciales es aún poco conclusiva, lo cual deja un claro papel para la política monetaria frente al riesgo sistémico.

Del mismo modo, podemos encontrar efectos colaterales de la política macroprudencial sobre la política monetaria. Si el objetivo prioritario de la política macroprudencial es garantizar el buen funcionamiento y resiliencia del sector financiero, implícitamente se está garantizando un buen mecanismo de transmisión monetaria, crucial para que la política monetaria alcance sus metas finales (Agénor \& Pereira da Silva, 2014). Del mismo modo, la política macroprudencial puede ayudar a la monetaria al reducir las disyuntivas en que se pueda encontrar, aumentando así su margen de maniobra. Dicho esto, surge la posibilidad de complementariedad entre política monetaria y macroprudencial, de manera que las acciones de una refuercen los objetivos de la otra.
Pero también pueden surgir conflictos. Un ejemplo sería cuando ante un fuerte crecimiento del crédito privado, la política macroprudencial reaccione endureciendo las condiciones de préstamo. Esta medida puede afectar al crecimiento y por ende a los precios. Si la política monetaria responde de manera acomodaticia reduciendo tipos de interés o aumentando la oferta de dinero, podría acabar retroalimentando el aumento del crédito inicial, entrando así ambas políticas en conflicto.

Dadas estas intersecciones entre ambas políticas, el debate está abierto en torno a la idoneidad de mantener dos políticas distintas o aunar todo en un único mandato. En este sentido, dados unos objetivos claros y bien diferenciados de cada una de las políticas, la teoría tradicional basada en la regla de Tinbergen (1952) sugiere que haya tantas políticas como objetivos a alcanzar, esto es, tantos instrumentos como objetivos (Mendoza, 2016) y que los instrumentos se asignen a aquellos objetivos para los que resulten más eficaces (Smets, 2014). Los partidarios de la regla de Tinbergen, abogan por una clara diferenciación de objetivos e instrumentos que potencien la credibilidad e independencia de las instituciones encargadas de defenderlos. De ahí que ante este debate sea necesario entender los canales a través de los cuales las políticas interfieren entre sí y con qué fuerza, para así identificar inequívocamente los objetivos a asignar a cada una de ellas.

\section{Interacciones entre política monetaria y macroprudencial}

Es importante partir de la premisa de que si cada política, tanto la monetaria como la macroprudencial, funcionara perfectamente, sin imperfecciones y enfocadas directamente en el objetivo que cada una tiene, no habría necesidad de preocuparse por posibles interacciones. El problema surge cuando las actuaciones de una política interfieren, en el sentido de que amplifican o pueden anular total o parcialmente los efectos 
TABLA 1

INTERACCIONES ENTRE LAS PRINCIPALES POLÍTICAS ECONÓMICAS

\begin{tabular}{|c|c|c|}
\hline Política (instrumento) & Objetivo & Meta final \\
\hline $\begin{array}{l}\text { Macroprudencial (ratios } \\
\text { LTV, colchones de capital } \\
\text { contracíclicos...) }\end{array}$ & Estabilidad financiera & \\
\hline $\begin{array}{l}\text { Monetaria (tipos de interés a } \\
\text { corto plazo) }\end{array}$ & Estabilidad de precios & $\begin{array}{l}\text { Crecimiento } \\
\text { sostenido } \\
\text { y precios estables }\end{array}$ \\
\hline $\begin{array}{l}\text { Fiscal y estructural (gasto } \\
\text { público, impuestos, deuda) }\end{array}$ & $\begin{array}{l}\text { Estabilidad del producto, } \\
\text { crecimiento equilibrado }\end{array}$ & \\
\hline
\end{tabular}

FUENTE: Elaboración a partir de Nier y Kang (2016) y Schoenmaker (2014).

de la otra. Es en este punto, en el que es necesario analizar dichas interacciones, la fuerza de las mismas y las posibles soluciones para garantizar el mejor funcionamiento de cada política.

\section{De la política monetaria a la política macroprudencial}

Como se ha mencionado antes, Nier y Kang (2016) es uno de los muchos trabajos que nos ofrece una clasificación detallada a la vez que concisa de los distintos canales de conexión entre política monetaria y macroprudencial. La Tabla 1 recoge las interacciones entre las principales políticas económicas.

Concretamente, estos autores los agrupan en función del factor económico donde se origina la inestabilidad que desencadena la respuesta de cada una de las políticas. De esta forma, podemos distinguir tres focos de inestabilidad.

Por un lado, tenemos el potencial de inestabilidad por parte de los balances de los prestatarios. En un contexto de tipos de interés bajos, en general resulta más barato pedir préstamos. Al mismo tiempo, si los precios de los activos son altos, aumenta el valor del colateral ofrecido en los contratos. Todo ello puede incitar a un aumento de la demanda de crédito.
En este mismo punto, funciona también el canal de impago. Cuando la política monetaria se vuelve expansiva, se reduce la probabilidad de impago de la mayoría de los prestatarios. Una menor probabilidad de impago se suele traducir en unos menores tipos de interés sobre préstamos para aquellos prestatarios más dudosos, permitiéndoles acceder a financiación de manera más fácil. Esta mejora de las condiciones de los préstamos afecta positivamente a la actividad económica, y acentúa aún más una posible expansión. Este efecto amplificador recoge la teoría del acelerador financiero, muy desarrollado en la literatura académica en particular por los trabajos de Bernanke, Gertler y Gilchrist (1996, 1999), así como Gertler y Gilchrist $(1993,1994)$.

La oferta de crédito también se ve afectada, a través del canal de riesgo. Unos tipos de interés bajos pueden aumentar la oferta de crédito, y el apalancamiento de los intermediarios financieros. En ocasiones, se relaja la monitorización y selección de proyectos, asumiendo así más riesgos en las inversiones. Este canal se retroalimenta ya que bajos tipos de interés reducen la probabilidad de impago, haciendo más atractivas las operaciones de préstamo con más riesgo (Adrian \& Shin, 2013) pues el riesgo que se percibe es menor. Estos efectos son más fuertes cuanto más tiempo dura la política monetaria acomodaticia. 
La política monetaria también tiene efectos en las posiciones de riesgo asumidas por los agentes ${ }^{9}$. Unos tipos de interés bajos pueden incentivar la búsqueda de activos con mayor rendimiento a cambio de asumir un riesgo mayor, el llamado search for yield (Rajan, 2005). Este canal es más susceptible de llevar a una crisis cuando la economía se encuentra más endeudada, ya que el margen para absorber impagos es menor ante un posible cambio en las condiciones monetarias.

Por último, también existen posibles inestabilidades a través de precios agregados tales como los precios de los activos y tipo de cambio. Como hemos mencionado antes, unos tipos de interés bajos pueden generar aumentos de precios de los activos ${ }^{10}$. En tanto en cuanto estos activos se utilicen como colateral, sus propietarios se podrán endeudar más, aumentando su vulnerabilidad ante un cambio en las condiciones monetarias, por ejemplo. Si bien este mecanismo ha sido muy fructífero desde el punto de vista teórico, la evidencia empírica sobre la dirección de causalidad entre política acomodaticia y precio de los activos no es del todo concluyente (Nier \& Kang, 2016).

Otro de los precios agregados que juega un papel importante como generador de inestabilidades financieras son los tipos de cambio. Esta variable es de especial relevancia para economías abiertas, tales como las economías emergentes $y$, en particular, en aquellas que piden prestado en moneda extranjera. Estas economías dependen de la política llevada a cabo por la

\footnotetext{
9 Véase Galati y Moessner (2013) y las referencias ahí incluidas sobre la evidencia empírica de esta relación.

10 Bien por el aumento de la demanda de activos más arriesgados, bien por una expansión o burbuja en algún sector específico, como, por ejemplo, el sector inmobiliario. En el caso del mercado de la vivienda los efectos son especialmente fuertes ya que este activo se utiliza como colateral para la demanda de nuevos préstamos, por lo que cambios bruscos en los precios de la vivienda afectarían tanto a la oferta como a la demanda de crédito, generando una alta inestabilidad financiera. A este respecto, España fue pionera en la utilización de instrumentos macroprudenciales con la implantación de las provisiones dinámicas o anticíclicas a principios de los años 2000 (Mencía \& Saurina, 2016).
}

autoridad monetaria de referencia ${ }^{11}$ en las transacciones financieras globales (Rey, 2013 y 2014). Un endurecimiento de la política monetaria estadounidense, por ejemplo, afectaría duramente a las condiciones crediticias de los países que se endeudan en dólares. Al mismo tiempo, un mayor tipo de interés extranjero ejercería presiones a la depreciación/devaluación de su moneda, aumentando la deuda del país. En la mayoría de las ocasiones se producen fuertes salidas de capitales (sudden stops), dificultando la financiación externa y aumentando su coste. Las consecuencias de estos cambios son mayores cuanto mayor es la deuda externa de estos países. Muchos países del sureste asiático y latinoamericanos se vieron en situaciones similares a lo largo de los años noventa, que llevaron a fuertes crisis financieras seguidas de crisis de deuda, las llamadas twin crises. De hecho, en estas economías el uso de políticas macroprudenciales fue muy anterior a su extensión entre las economías avanzadas, con el objetivo de reducir su vulnerabilidad a sufrir fuertes salidas de capitales y aumentos de sus posiciones deudoras por cambios en las condiciones monetarias de la moneda en que pedían prestado ${ }^{12}$.

\section{De política macroprudencial a política monetaria}

De la misma manera que la política monetaria puede tener efectos sobre la estabilidad financiera, la política macroprudencial puede acabar afectando a la estabilidad económica y, en particular, a la estabilidad de precios.

Por un lado, desde el punto de vista de los prestamistas, una política macroprudencial restrictiva puede reducir la oferta de crédito, limitando la cantidad de proyectos e inversiones que se puedan llevar a cabo. Esta menor oferta de posibilidades de financiación

\footnotetext{
11 Por excelencia la Reserva Federal, aunque el Banco Central Europeo está cobrando cada vez un mayor papel en este sentido (Cerutti et al., 2014).

12 Mendoza (2016) analiza los retos y promesas de la política macroprudencial. Véanse las referencias ahí incluidas sobre diversos análisis aplicados a países emergentes.
} 
tiene efectos negativos sobre el crecimiento de la economía como sobre los precios, afectando de lleno a las competencias propias de la política monetaria.

Por otro lado, una expansión monetaria que conlleve un aumento en los precios de los activos puede generar un efecto riqueza en los prestatarios que les incentive a pedir más prestado (Jordà et al., 2017). En este caso, ante una política monetaria laxa que incentive la demanda de crédito, la política macroprudencial dirigida a los prestatarios, con el fin de evitar posiciones demasiado endeudadas de estos agentes, puede contrarrestar los efectos expansivos de dicha política monetaria. En este caso también acabaría teniendo efectos sobre los precios y la estabilidad económica. $Y$ más aún, podría impedir a la política monetaria alcanzar sus objetivos.

Por último, los tipos de interés sobre préstamos finales también pueden verse afectados por la regulación financiera, pudiendo reducir los efectos buscados de una determinada política monetaria. Por ejemplo, ante un aumento de los requerimientos de capital, las entidades financieras pueden decidir repercutir ese mayor coste en el precio al que conceden créditos.

\section{¿Políticas coordinadas o independientes?}

En este contexto surge el debate sobre si es necesario coordinar política monetaria y macroprudencial, así como sobre la institución encargada de las dos o de cada una de ellas. La cuestión es mucho más relevante si tenemos en cuenta que el diseño de la política macroprudencial está dirigido principalmente al sector financiero bancario. En un escenario económico en el que el sector financiero no bancario cobra cada vez mayor relevancia, es clave la coordinación entre política macroprudencial y monetaria con el fin de limitar el flujo de préstamos del sector bancario (más regulado) hacia el no bancario o en la sombra (Gebauer \& Mazelis, 2020). El diseño de la política monetaria es crucial para afectar a este último tipo de entidades, ya que su actividad se ve sobre todo alterada por variaciones en el tipo de interés, más que por medidas macroprudenciales propiamente dichas. En este sentido, dicho problema sugiere la creación de instrumentos o bien dirigidos a ambos sectores o bien lo suficientemente generales como para que regulen las actividades financieras independientemente del agente que las lleve a cabo.

Es importante destacar que el consenso es que la política monetaria debería bastar en ausencia de fricciones financieras. Sin embargo, con imperfecciones en los mercados financieros surge un papel crucial de la política macroprudencial. Desde la crisis financiera global han proliferado los modelos teóricos para el análisis de los efectos de la política macroprudencial y sus interacciones con la política monetaria. Estos modelos introducen en ocasiones fricciones financieras por el lado de los prestatarios y más recientemente por el lado de los prestamistas. La Tabla 2 recoge algunos de los principales y más recientes modelos.

De todo este análisis se deriva que no existe un único marco ni un único instrumento para la aplicación de la política macroprudencial. En la mayoría de los casos, la idoneidad de coordinar ambas políticas depende de la perturbación que esté afectando a la economía y de las fricciones que haya. Y más aún, en estos modelos se supone que las políticas macroprudenciales están perfectamente enfocadas en su objetivo, cuando en realidad pueden apuntar a la variable errónea, estar mal calibradas o presentar retardos en su aplicación, lo cual dificulta mucho la evaluación real de sus efectos.

La literatura académica sigue avanzando, incorporando nuevos factores y externalidades para analizar la optimalidad de combinar políticas monetarias y macroprudenciales. Así, Farhi y Werning (2016) plantean la existencia de externalidades por el lado de la demanda agregada, a diferencia del enfoque tradicional que supone externalidades pecuniarias. Los autores analizan las políticas monetaria y macroprudencial óptimas en varios escenarios, desde economías cerradas a pequeñas economías abiertas. 
TABLA 2

\section{ALGUNOS ESTUDIOS RECIENTES SOBRE INTERACCIÓN ENTRE POLIITICA MONETARIA Y MACROPRUDENCIAL}

\section{Artículo}

Evaluación de políticas
Instrumento de política monetaria

Instrumento de política macroprudencial
Angelini et al. (2014)

Angeloni y Faia (2013)

Bailliu et al. (2015)

Christensen et al. (2011)

Collard et al. (2017)

Darracq et al. (2011)

De Paoli y Paustian (2017)

Kannan, Rabanal y Scott (2009)

\section{con}

Incluir la política macroprudencial como instrumento adicional supone una mejora ante shocks financieros.

Si se pueden identificar bien los shocks, la combinación óptima de políticas depende del shock que afecte a la economía.

Rubio y Carrasco-Gallego (2015) Regla de Tinbergen. Óptimo si ambas políticas están en vigor, cada una con sus propios objetivos.

Rubio y Yao (2019)

Ganancias de cooperación entre políticas, mayores ante shocks financieros que de oferta.

Combinación óptima de ambas políticas con la política monetaria leaning against the wind.

Coordinación entre políticas con la política monetaria

La política macroprudencial estabiliza la macroeconomía sobre todo ante shocks financieros.

Cooperación entre políticas siempre supone una ganancia.

La inestabilidad financiera surge del tipo de los contratos, no de su cuantía.

Regla de Tinbergen. Con instrumentos de política que afectan a márgenes distintos, el principio de separación se cumple politica monetaria y macroprudencial.

La política macroprudencial estabiliza la macroeconomía sobre todo ante shocks financieros, aliviando la presión sobre la política monetaria. Fuertes complementariedades.

Solo si la política macroprudencial no es cíclica, es óptimo para la política monetaria reaccionar a variables financieras, con el coste de mayor volatilidad de la inflación.

il las fricciones afectan a todos los factores productivos por igual, la política macroprudencial se convierte en un instrumento clave, con o sin cooperación.

No es necesario cooperación entre políticas si la macroprudencial es la política líder.

La política monetaria que reacciona a variables financieras consigue la estabilización macroeconómica.

Con tipos de interés altos, los resultados de la cooperación
Regla de Taylor.

Regla de Taylor.

Regla de Taylor. LTV. Impuesto que afecta al capital de los bancos.

ratio similar a una regla de Taylor que responde al producto y a los precios de la vivienda.

Requerimientos de capital contracíclicos.

capital bancario.

Impuesto sobre préstamos. capital contracíclicos

Requerimientos de capital.

Requerimientos de capital contracíclicos y sensibles al riesgo.

Impuestos sobre préstamos.
Requerimientos de (si esta es factible) entre políticas asemejan los de la regla de Tinbergen.

Cuando los tipos de interés son bajos (incluso en el límite cero), la política macroprudencial es clave, incluso reaccionando al producto. 


\section{TABLA 2 (Continuación)}

\section{ALGUNOS ESTUDIOS RECIENTES SOBRE INTERACCIÓN ENTRE POLÍTICA MONETARIA Y MACROPRUDENCIAL}

\begin{tabular}{llc}
\hline Artículo & Evaluación de políticas & $\begin{array}{r}\text { Instrumento de } \\
\text { política monetaria }\end{array}$ \\
\hline
\end{tabular}

\section{Silvo (2019)}

Tayler y Zilberman (2016)

Van der Ghote (2018)
El uso de reglas simples sugiere que es óptimo que la política monetaria responda a los shocks financieros.

Cooperación entre políticas es óptimo ante shocks de oferta.

Si los shocks son financieros, el principio de separación aplica.

Cooperación entre ambas políticas es mejor para la estabilidad de precios, con la política monetaria reaccionando a los márgenes de crédito.

El uso de la política macroprudencial afecta al grado de agresividad de la política monetaria frente a la inflación.

La política monetaria contribuye a la estabilidad financiera.

Cooperación entre políticas implica una política macroprudencial menos agresiva, pero a cambio de un moderado aumento de la volatilidad de la inflación.
Regla de Taylor.

Requerimientos de capital contracíclicos.
Regla de Taylor.

Regla de Taylor.

Regla de Taylor monetaria.

Mayores ganancias de introducir política macroprudencial específica a cada país ante shocks asimétricos.
Dehmej y Gambacorta (2019)

de Blas (2020)

Quint y Rabanal (2014)
La política macroprudencial contribuye ahí donde la monetaria no puede actuar debido a shocks asimétricos.

En el caso de shocks simétricos, la política macroprudencial reduce la presión sobre la monetaria.

Papel relevante del mercado interbancario.

La política monetaria que reacciona a variables financieras consigue estabilizar la economía.

La política macroprudencial eficaz para reducir los diferenciales de los márgenes crediticios entre países.

Papel complementario entre política macroprudencial y monetaria.

Efectos diferenciales de la política macroprudencial entre prestatarios y ahorradores dependiendo del shock.
Regla de Taylor. Margen de crédito.

Regla de Taylor. Demanda de crédito: impuesto sobre préstamos.

Oferta de crédito: requerimientos de capital (específicos de país y a nivel de la unión monetaria).

Regla de Taylor.

Contracíclico respondiendo a crecimiento del crédito nominal y la ratio de crédito sobre PIB

Escenarios: instrumentos comunes a la unión vs. específicos a cada país.

NOTAS: La tabla muestra algunos de los múltiples modelos de equilibrio general dinámico y estocásticos (DSGE, por sus siglas en inglés) que incluyen fricciones financieras por el lado de los prestamistas y/o de los prestatarios.

FUENTE: Elaboración propia. 
Uno de los puntos de análisis es cómo ambas políticas pueden potenciar sus efectos mutuamente (FMI, 2013). Una política macroprudencial efectiva que tenga unos objetivos claros y unos instrumentos bien definidos, alcanzando así la estabilidad financiera, permitiría a la política monetaria centrarse en su objetivo de estabilidad de precios, incluso sin necesidad de llevar a cabo medidas muy agresivas para alcanzar los mismos objetivos. Este refuerzo entre políticas también funciona positivamente en términos de la credibilidad de las instituciones encargadas. Con unos objetivos únicos y claros, es más fácil mantener la credibilidad y evitar interferencias entre ellas. Por último, la política macroprudencial puede reducir los problemas de riesgo moral ante políticas monetarias laxas, permitiendo así mayor margen de maniobra a esta última.

Basso y Costain (2016) se hacen eco de este debate. Por un lado, los autores plantean el punto de vista proindependencia de las políticas, según el cual los tipos de interés no son un buen instrumento para reducir la deuda y, por lo tanto, para conseguir estabilidad financiera ${ }^{13}$. Pero incluso si pudiera alcanzarla, pondría en juego su objetivo de estabilidad de precios, por lo que siempre que hubiera una política macroprudencial disponible, la política monetaria no debería entrar en cuestiones de riesgo sistémico (Svensson, 2014; Weidmann, 2014). Por otro lado, presentan el punto de vista procolaborativo según el cual el principal canal entre política monetaria y estabilidad financiera es vía la asunción de riesgo por parte de los agentes, especialmente fomentada por largos períodos de tipos bajos. Dada la relevancia del canal de riesgo para la dinámica de los ciclos financieros, este enfoque aboga por una política monetaria que incorpore objetivos de estabilidad financiera en su estrategia (Stein, 2011; Borio \& White, 2003).

\footnotetext{
13 De hecho, Basso y Costain (2016) destacan que utilizar los tipos de interés para reducir la deuda puede no ser tan efectivo: si unos tipos de interés más altos reducen la inflación, esto puede dar lugar a un aumento de la deuda real mucho más rápidamente que a una reducción de la misma (debt-deflation).
}

En otro artículo relevante, Smets (2014) argumenta que la política monetaria debería tener en cuenta la estabilidad financiera en función de tres aspectos: la efectividad de la política macroprudencial, los efectos de los tipos de interés sobre la estabilidad financiera y de hasta qué punto se vería cuestionado el objetivo de inflación. Tras una revisión de la teoría y la evidencia, el autor aboga por mantener una separación de objetivos entre la política monetaria y la macroprudencial en la línea de la regla de Tinbergen. Sin embargo, plantea la posibilidad de que la política monetaria esté vigilante para intervenir en caso de inestabilidad financiera. Entonces, ¿debería la política monetaria incluir entre sus objetivos el de estabilidad financiera? El debate se canaliza a través de tres vías. Por un lado, se plantea el «consenso modificado de Jackson Hole» ${ }^{14}$ según el cual la política macroprudencial debe presentar un marco efectivo y creíble para mantener la estabilidad financiera. Esto permitiría a la política monetaria centrarse en la estabilidad de precios, pero teniendo en cuenta los efectos de factores financieros sobre su mecanismo de transmisión (surge así la necesidad de financial monitoring). Este enfoque parte de que se pueden separar fácilmente los objetivos, instrumentos y mecanismos de transmisión entre ambas políticas, que los efectos de las interacciones son limitados y que el tipo de interés no es eficaz para evitar desequilibrios financieros.

La segunda vía consiste en reivindicar el que la política monetaria haga frente a las fluctuaciones financieras (leaning against the wind). La idea subyacente es que la política macroprudencial no puede controlar del todo el

\footnotetext{
14 La conferencia de Jackson Hole (Reserva Federal de Kansas City) reúne desde 1978 a banqueros centrales, ministros de Finanzas, destacados profesionales académicos y financieros con el objetivo de discutir cuestiones económicas actuales y de política económica. De esta conferencia surgió el llamado consenso de Jackson Hole según el cual la política monetaria solo debe preocuparse por la estabilidad financiera en tanto en cuanto esta amenace la estabilidad de precios y de la economía. Para una descripción de la evolución en materia de estrategias de política monetaria desde la Gran Moderación véase Berganza et al. (2014).
} 
ciclo financiero. Además, la política monetaria, al fomentar posiciones de riesgo, puede alterar el mecanismo de transmisión monetaria, con los consiguientes efectos sobre la estabilidad de precios. Por ello, según esta vía, la política monetaria debería incluir como objetivo secundario la estabilidad financiera, y abogar por un objetivo de inflación flexible que permita incluir aspectos de estabilidad financiera y así contrarrestar esa posible inestabilidad (Smets, 2014). Los modelos desarrollados para analizar esta vía las consideran imperfecciones de crédito y motivan que la política monetaria responda en cierto modo a aspectos financieros, pero sin perder la estabilidad de precios como objetivo prioritario. Sin embargo, los ciclos financieros muestran frecuencias distintas de los ciclos económicos, lo cual puede entorpecer el manejo de la política monetaria. Por su parte, la idoneidad de la estrategia leaning against the wind también está en cuestión, ya que hay poca evidencia de los beneficios de dicha política (Svensson, 2018), además de que los costes parecen sobrepasar los beneficios (FMI, 2015). Esta posición también se encuentra en otras discusiones de la literatura como recogen Berganza et al. (2014).

La última vía que se propone en este debate es aquella según la cual la «estabilidad financiera significa estabilidad de precios». Es decir, mantener como objetivo prioritario la estabilidad financiera ya que ello llevaría a la estabilidad de precios. Este punto de vista es ampliamente rebatido por aquellos que afirman que la crisis financiera puso de manifiesto que la estabilidad de precios no lleva a la estabilidad financiera (FMI, 2013; Berganza et al., 2014). En este sentido, Brunnermeier y Sannikov (2014), presentan un modelo que permite analizar cómo interaccionan la estabilidad de precios y la financiera. La idea es que un sistema financiero saneado «determina el grado de creación de dinero y pone precio al riesgo de la economía». En este contexto, el papel de la política monetaria es de redistribuir la riqueza entre los agentes, reduciendo el riesgo de amplificación de las imperfecciones financieras y alcanzar así la estabilidad de precios. Sin embargo, esta visión lleva consigo cuestiones de inconsistencia temporal de las políticas por el peligro del dominio financiero ${ }^{15}$, por lo que la coordinación entre ambas es crucial.

\section{Argumentos en contra de coordinar política macroprudencial con política monetaria}

De concluir que cierta coordinación entre la política macroprudencial y la monetaria puede ser en ocasiones deseable, esta no queda exenta de posibles complicaciones. ¿Quién debe encargarse de la política macroprudencial? ¿Debe recaer sobre el banco central o es más deseable delegarla a otra institución independiente?

Una de las principales amenazas se encuentra en la pérdida de credibilidad por parte del banco central. El éxito de los objetivos de inflación desde los años noventa radica en gran parte en haber sido dirigidos por unos bancos centrales independientes, que se han forjado una credibilidad antiinflacionista que los mercados y demás agentes han creído. Atribuir o asumir objetivos de estabilidad financiera que en ocasiones pueden ir en contra de la estabilidad de precios supone minar la confianza en esos bancos centrales.

Por otro lado, expandir los objetivos de la política monetaria para incluir aquellos de estabilidad financiera puede, como hemos mencionado antes, dar lugar al dominio financiero, poniendo así en duda la independencia de los bancos centrales.

Todo ello genera serios problemas de inconsistencia temporal (Smets, 2014). Un banco central que se compromete a la estabilidad de precios, pero contempla también objetivos de estabilidad financiera, puede, llegado el momento, relegar uno de los objetivos a favor del otro. Como en el caso de la política fiscal y monetaria, los problemas de inconsistencia temporal se pueden resolver "atando las manos» de la institución encargada de ellos. Es por ello, que quienes ven

\footnotetext{
15 Por dominio financiero entendemos la posibilidad de que los desarrollos financieros acorralen a los bancos centrales para que lleven a cabo determinadas políticas que limiten su lucha antiinflacionista.
} 
serias amenazas de inconsistencia temporal que puedan llevar a la pérdida de credibilidad de los bancos centrales abogan por separar el conducto de ambas políticas. De esta manera, deberían crearse instituciones independientes con reglas y objetivos claros en el ámbito de la política macroprudencial.

Mendoza (2016) plantea además otras posibles complicaciones en el diseño y la aplicación de la política macroprudencial que hay que tener en cuenta. Por un lado, su complejidad. Es importante saber cuándo actuar, y el debate está abierto. ¿Hay que reaccionar ante posibles burbujas de precios $u$ otros desequilibrios financieros? ¿Cómo identificamos si de verdad suponen una amenaza para la estabilidad financiera? ¿Quién va a estropear la fiesta cuando las cosas «van bien»? Intentar frenar desequilibrios financieros, sobre todo en épocas de auge, puede ser ex ante costoso. Pero esperar a que la situación estalle, puede ser ex post ineficiente, sobre todo porque una vez en fase de caída tal vez no se consiga atenuar los efectos buscados, o tal vez sea demasiado tarde para evitar la transmisión de los efectos a toda la economía (es decir, riesgo sistémico). Por otro lado, la implementación de la política macroprudencial puede implicar retardos, así como dificultad a la hora de cambiar los instrumentos. Todo ello pone en cuestión la eficacia de dicha política, pudiendo generar los efectos contrarios a los deseados.

\section{Factores adicionales - restricciones sobre las políticas}

Como hemos visto, en ocasiones la política monetaria no es capaz de conseguir la estabilidad financiera. Esto ocurre, por ejemplo, cuando la inestabilidad surge por motivos distintos de los ligados a la cantidad de liquidez en el sistema, que es donde la política monetaria puede actuar directa y más eficientemente. En este apartado, analizaremos factores adicionales a tener en cuenta a la hora de aplicar la política monetaria y macroprudencial y que dependen en gran parte de las restricciones que haya sobre ellas.

\section{Uniones monetarias}

Un caso especial es el de las uniones monetarias (Darracq et al., 2019; de Blas, 2020). Cuando la política monetaria se encuentra restringida a nivel nacional como en uniones monetarias, la política macroprudencial cobra mayor relevancia (FMI, 2013). Pero al debate desarrollado anteriormente sobre el conducto de cada una de estas políticas se añade la disyuntiva de a qué nivel debe llevarse a cabo la política macroprudencial, si a nivel nacional o a nivel de la Unión (Dehmej \& Gambacorta, 2019). Los desarrollos teóricos sobre estos aspectos son aún recientes y el debate está muy abierto. Por un lado, factores que influyen sobre el carácter nacional o supranacional de la autoridad macroprudencial incluyen el grado de integración financiera entre los países de la unión, la existencia de normas homogéneas de política prudencial (tanto a nivel macro como micro), la estructura del sistema financiero de cada país (muy basado en bancos pequeños, más desarrollado financieramente, etc.), así como el mecanismo de transmisión predominante en cada país. En el caso de la eurozona, el BCE/ESRB tiene la supervisión de las entidades financieras nacionales que suponen riesgo sistémico (este riesgo se va evaluando periódicamente), y deja la autoridad supervisora para las demás entidades a los bancos nacionales.

Por otro lado, la crisis de deuda soberana que afectó a Europa entre 2011-2013 puso de manifiesto la debilidad de una unión monetaria sin una unión fiscal. Sobre todo, por la retroalimentación que surgió en algunos países de la zona euro entre riesgo soberano y riesgo bancario. En una unión monetaria que carece de unión fiscal, es necesario disponer de instrumentos flexibles a nivel regional con los que poder hacer frente a shocks asimétricos, abriendo la puerta al desarrollo de políticas macroprudenciales. Al mismo tiempo, una mayor homogeneización fiscal conllevaría una mayor armonización macroprudencial. Esta necesidad de instrumentos flexibles es incluso mayor si la política fiscal está al mismo 
tiempo restringida por fuertes reglas de déficit y ratios deuda pública-PIB como las impuestas por el Pacto de Estabilidad y Crecimiento en la eurozona.

\section{Tipos de interés bajos y políticas monetarias no convencionales}

Hemos identificado una de las interacciones entre la política monetaria y la macroprudencial como la situación en que bajos tipos de interés fomentan posiciones de riesgo tanto entre prestamistas (financieros o no) como prestatarios. Esta interacción puede plantear mayores problemas en un contexto de tipos de interés bajos e incluso negativos durante largos períodos de tiempo, como en la actualidad (Constâncio, 2017). En un discurso reciente, el vicepresidente del Consejo de Supervisión del Banco Central Europeo, Yves Mersch (2020), mencionaba la necesidad de vigilar los efectos colaterales de una política monetaria como la actualmente llevada a cabo por el BCE a este respecto, y la necesidad de recalibrar y adecuar el tono de las medidas monetarias para reducir tales riesgos. En este sentido, cada vez son más las voces dentro del seno de la eurozona que abogan por considerar aspectos de estabilidad financiera en las decisiones de política monetaria, si bien estas voces destacan que la estrategia en dos pilares del BCE ya permite tener dichos aspectos en consideración (Smets, 2014; Mersch, 2020).

Es por ello que en el escenario de tipos de interés bajos y políticas monetarias no convencionales es preciso una fuerte colaboración entre las dos políticas. Cuando el tipo de interés real es negativo, el banco central se ve obligado a intervenir continuamente en los mercados financieros y a mantener los tipos en niveles bajos. Estas intervenciones pueden generar inestabilidad financiera, y es aquí donde la política macroprudencial más enfocada en los distintos segmentos del mercado, puede colaborar para contrarrestar esa fuente de inestabilidad. De esta forma, la efectividad de ambas políticas se vería reforzada. Esta fuente de colaboración también surge, por ejemplo, a través de las facilidades de crédito que pueden permitir a las entidades financieras relajar sus restricciones de liquidez (y por lo tanto, reducir los riesgos de inestabilidad) al tiempo que proporcionan crédito.

\section{El dilema sobre los precios de la vivienda}

En sí, la asunción de riesgo por parte de los agentes económicos no es mala, el problema aparece cuando ese riesgo es excesivo y puede poner en jaque la estabilidad de todo el sistema financiero. Períodos de bajos tipos de interés coinciden con precios altos de los activos (como los precios de la vivienda y de otros activos financieros). Ante los bajos rendimientos de activos seguros, la demanda de los inversores se redirige hacia otros activos, a cambio de más riesgo, y este exceso de demanda hace aumentar su precio ${ }^{16}$. En este contexto, la política macroprudencial es clave, en particular si los tipos de interés bajos se mantienen durante mucho tiempo alcanzando su nivel mínimo efectivo ${ }^{17}$. Sin embargo, no es seguro que ella sola pueda evitar la generación de desequilibrios. Factores adicionales, tales como fuentes de financiación internacional para el sector financiero doméstico o la banca en la sombra, pueden aliviar las posibles medidas contractivas que se lleven a cabo, tanto a nivel de política monetaria como macroprudencial, reduciendo así sus efectos estabilizadores. Un activo que genera especial preocupación por sus efectos más allá de la estabilidad financiera es el precio de la vivienda. Los efectos de la crisis financiera

\footnotetext{
16 Una serie de estudios documenta que la senda alcista de los precios de los activos en los últimos años coincide con una tendencia decreciente de los tipos de interés. Esta senda decreciente se puede explicar por cambios seculares, como, por ejemplo, aumento de la edad de la población, distribución de la renta, aumento de las tasas de ahorro en países emergentes y un aumento general de la aversión al riesgo entre otros (véanse las referencias citadas en Mersch, 2020).

${ }_{17}$ Algunos trabajos que analizan la interacción entre política monetaria y macroprudencial en períodos de tipos de interés cero o políticas no convencionales incluyen Rubio y Yao (2019) y las referencias ahí citadas, entre otros.
} 
global fueron especialmente perniciosos en países que habían experimentado una burbuja inmobiliaria, tanto en términos cíclicos como de distribución de la renta, como, por ejemplo, España, Reino Unido o Irlanda.

Algunos bancos centrales, como el de Estados Unidos, Japón, Suecia o Noruega, incluyen en sus índices de precios de referencia el de la vivienda ocupada por su propietario. No es el caso para el Banco Central Europeo, donde el índice armonizado de precios al consumo (IAPC) no incluye tales costes, si bien casi dos tercios de los habitantes de la eurozona son propietarios de una vivienda. En enero de 2020, el BCE empezó un período de revisión de su estrategia de política monetaria, incluyendo cuestiones como si es necesario considerar o no el precio de la vivienda y el reanclamiento de las expectativas de inflación (The Economist, 2020).

Considerar este precio en el índice de referencia para la inflación no es tan sencillo ya que tiene ventajas e inconvenientes. Por un lado, serviría para integrar en los objetivos de política monetaria un precio fuertemente ligado a la estabilidad financiera. Al mismo tiempo, el precio de la vivienda presenta una mayor volatilidad cíclica. Si la política monetaria respondiera a sus variaciones, es decir, fuera más activista, las variaciones derivadas de los tipos de interés podrían generar un impacto negativo en términos de bienestar. Por otro, su inclusión en el IAPC hoy en día aumentaría las tasas de inflación observadas en la eurozona (Mersch, 2020) afectando, por lo tanto, al tono de la política monetaria. También influiría en la percepción que tengan los individuos sobre el nivel de inflación, pudiendo cuestionarse entonces la necesidad de medidas no convencionales. Si, además, las expectativas de inflación están desancladas, la política monetaria tiene un obstáculo más para su efectividad. Además, el IAPC es por definición un índice de precios de bienes de consumo, no incluye activos. La vivienda es un activo que proporciona servicios a los individuos y aislar el coste de estos servicios es complicado. Por otro lado, tampoco es fácil desligarlo del precio del suelo, un factor sujeto a fuertes fluctuaciones en su precio y que, de nuevo, no se ajusta a la definición del IAPC.

\section{Retos para el análisis macroeconómico}

Los modelos económicos nos muestran que el diseño idóneo de políticas monetaria y macroprudencial, tanto si deben ser coordinadas o no, depende en gran medida de los supuestos subyacentes. Es por ello que se necesitan más análisis. Por ejemplo, Farhi y Werning (2020) estudian el diseño óptimo de estas políticas cuando las expectativas no son racionales y surge una externalidad dual: externalidades por el lado de la demanda y por el lado de expectativas endógenas. Las primeras externalidades se dan cuando los prestatarios no internalizan las consecuencias que su excesivo endeudamiento puede tener en ahondar la caída de la demanda cuando se llegue a una recesión. La externalidad de creencias endógenas recoge que unos prestatarios demasiado optimistas no internalizan el hecho de que al tomar prestado demasiado su bienestar se reduce. Como resultado, las combinaciones óptimas de política monetaria y macroprudencial varían cuando esta externalidad dual está presente. En particular, los autores encuentran que la política macroprudencial puede no ser suficiente para conseguir la estabilidad financiera cuando las expectativas que forman los agentes no son racionales, sugiriendo así un papel activo de la política monetaria.

Este tipo de trabajos nos mantiene en el debate entre independencia y coordinación de las políticas y pone de manifiesto la necesidad de más estudios para resolverlo.

\section{Diseños institucionales en distintos países}

El objetivo de este apartado es analizar los retos institucionales y de gobernanza que se han planteado en la implementación de políticas monetaria y macroprudencial.

De la experiencia internacional se desprenden dos estrategias institucionales distintas. Algunos países han delegado el manejo de la política macroprudencial a 
TABLA 3

\section{DISEÑO INSTITUCIONAL — EXPERIENCIA INTERNACIONAL}

Muestra de países donde el papel de la autoridad macroprudencial y el de la autoridad designada coinciden en la misma institución

Banco Central

Otro

Bélgica, Bulgaria, Chipre, República Checa, Estonia, Grecia,

Irlanda, Lituania, Malta, Portugal, Eslovaquia, Reino Unido,

Nueva Zelanda, Hong Kong, Singapur

Bulgaria, Finlandia, Francia, Noruega, Polonia, Rumanía, Suecia, Canadá, Australia, Corea del Sur, Suiza

Estados miembros del EEA donde el papel de la autoridad macroprudencial y el de la autoridad designada son distintas

Autoridad designada*

Autoridad macroprudencial**

Banco Central

Otro
Banco Central

Italia

Croacia, Hungría, Islandia, Luxemburgo, Países Bajos, Eslovenia, España
Otro

Islandia, Letonia, Luxemburgo

Austria, Dinamarca, Alemania, Liechtenstein

NOTAS: *Autoridad designada: establecida de acuerdo con el artículo 136 de la Directiva 2013/36/EU (CRD IV). Esta autoridad designada está a cargo de la implementación de las medidas de CRD y CRR. ${ }^{* *}$ Autoridad macroprudencial: establecida de acuerdo con la Recomendación ESRB/2011/3. Esta autoridad puede aconsejar, guiar y recomendar sobre cuestiones de política macroprudencial a otras autoridades.

FUENTE: Elaborado a partir de «List of national macroprudential authorities and national designated authorities in EEA member states», ESRB, 25/11/19, FMI Macroprudential policy database (2018).

una entidad independiente. En este caso, esta entidad puede encontrarse dentro del banco central o fuera. Otros han asignado al banco central la competencia sobre política macroprudencial. La Tabla 3 recoge una clasificación posible de la experiencia internacional al respecto a fecha de redacción de este artículo.

La Tabla 3 recoge la distribución de países en función del diseño institucional que han elegido para la política macroprudencial. El panel superior incluye aquellos países que han designado una única entidad para gestionar todos los aspectos macroprudenciales. El panel inferior recoge aquellos países que asignan al banco central el control de los requerimientos de capital, y a otra entidad (ya sea dentro del propio banco central, o un consejo externo o autoridad independiente) la elaboración de recomendaciones y manejo de otros instrumentos macroprudenciales. Con la excepción de tres países (Bulgaria, Islandia y Luxemburgo), las competencias están bien delimitadas. Es de destacar la heterogeneidad de esquemas que reina en el seno de la eurozona, lo cual propicia una gran variedad de escenarios a analizar, además de suponer un reto para el manejo de una política monetaria común.

La evidencia muestra que los factores institucionales parecen influir en la efectividad de las medidas de política macroprudencial (Edge \& Liang, 2019 y 2020). Por un lado, la existencia de autoridades tales como comités de estabilidad financiera ${ }^{18}$ parece aumentar la probabilidad de adoptar medidas macroprudenciales en la línea de Basilea III. Por otro lado, es importante que además estos comités estén dotados de buenos instrumentos y procedimientos para llevar a cabo dichas medidas macroprudenciales.

Dada la posible existencia de interacciones entre política monetaria y macroprudencial, los aspectos mencionados anteriormente de independencia, credibilidad y

\footnotetext{
18 Financial stability committees (FSCs) por sus siglas en inglés.
} 
definición de objetivos hacen necesario un diseño institucional que garantice que dichas interacciones no entorpezcan el manejo de cada una de las políticas. Una definición clara de los objetivos, una rendición de cuentas por parte de las autoridades competentes y la garantía de que se puede llevar a cabo una coordinación entre las políticas son aspectos clave.

El debate en este sentido se asemeja mucho al que hubo en su momento sobre el manejo de la política monetaria y la política fiscal, donde el diseño de políticas independientes se impuso. En el caso de la política macroprudencial el debate sigue aún abierto.

\section{Conclusiones}

El objetivo de este artículo es revisar las interacciones entre política monetaria y macroprudencial, así como los posibles retos que dichas interconexiones pueden plantear para un manejo eficiente de cada una de las políticas.

En determinadas ocasiones la coordinación puede ser deseable, pero siempre que la política monetaria mantenga un claro objetivo de estabilidad de precios y que sea la macroprudencial la que ayude donde la monetaria no llegue. Esta colaboración parece ser más eficaz si ambas políticas se llevan a cabo por autoridades independientes, con garantías que distingan entre las dos funciones de las políticas a través de la toma de decisiones separada, objetivos e instrumentos claros, rendición de cuentas y fuertes estructuras de comunicación (FMI, 2013).

En el contexto actual de tipos de interés bajos y políticas monetarias no convencionales, urge más la coordinación entre la política monetaria y la macroprudencial. De esta manera se puede garantizar la efectividad de la primera sin poner en juego la estabilidad financiera. La discusión sobre la combinación óptima de ambas políticas está más abierta que nunca y, sin duda, el desarrollo de nuevos modelos y la evaluación de la evidencia empírica más reciente nos ayudará a arrojar más luz sobre este debate.

\section{Referencias bibliográficas}

Adrian, T. \& Shin, H. S. (2013). Procyclical leverage and valueat-risk. The Review of Financial Studies, 27(2), 373-403.

Agénor, P. R. \& Pereira da Silva, L. A. (2014). Macroprudential regulation and the monetary transmission mechanism. Journal of Financial Stability, 13, 44-63.

Aguilar, P., Arce, Ó., Hurtado, S., Martínez-Martín, J., Nuño, G. \& Thomas, C. (2020). La respuesta de la política monetaria del Banco Central Europeo frente a la crisis del COVID-19. Documentos ocasionales del Banco de España, 26, 1-26.

Angelini, P., Neri, S. \& Panetta, F. (2014). The interaction between capital requirements and monetary policy. Journal of Money, Credit and Banking, 46(6), 1073-1112.

Angeloni, I. \& Faia, E. (2013). Capital regulation and monetary policy with fragile banks. Journal of Monetary Economics, 60(3), 311-324.

Bailliu, J., Meh, C. \& Zhang, Y. (2015). Macroprudential rules and monetary policy when financial frictions matter. Economic Modelling, 50, 148-161.

Banco Central Europeo (2017). Financial cycles and the macroeconomy. ECB Economic Bulletin, 1, 24-27.

Banco de Inglaterra (2011). Instruments of macroprudential policy. Discussion paper.

Basso, H. S. \& Costain, J. S. (2016). Macroprudential theory: Advances and challenges. Documentos Ocasionales del Banco de España N. ${ }^{\circ} 1604$.

Berganza, J. C., Hernando, I. \& Vallés, J. J. (2014). Los desafíos para la política monetaria en las economías avanzadas tras la gran recesión. Documentos Ocasionales del Banco de España N. ${ }^{\circ} 1404$.

Bernanke, B. S., Gertler, M. \& Gilchrist, S. (1996). The financial accelerator and the flight to quality. The Review of Economics and Statistics, 78(1), 1-15.

Bernanke, B. S., Gertler, M. \& Gilchrist, S. (1999). The financial accelerator in a quantitative business cycle framework. In J. B. Taylor \& M. Woodford (eds.), Handbook of Macroeconomics (pp. 1341-1393).

Bernie, J., Dalitz, L., Ejsing, J., Grothe, M., Manganelli, S., Monar, F., Sahel, B., Susec, M., Tapking, J. \& Vong, T. (2011). The impact of the Eurosystem's covered bond purchase programme on the primary and secondary markets. ECB Occasional Paper No. 122.

Blanchard, O. J., Dell'Ariccia, G. \& Mauro, P. (2013). Rethinking macro policy II: getting granular. IMF Staff discussion note SDN/13/03.

Borio, C. (2012). The financial cycle and macroeconomics: What have we learnt? BIS Working Paper No. 395.

Borio, C. \& White, W. R. (2003). Whither monetary and financial stability: the implications of evolving policy regimes. 
Proceedings - Economic Policy Symposium - Jackson Hole, 131-211.

Brunnermeier, M. K. \& Sannikov, Y. (2014). A Macroeconomic Model with a Financial Sector. American Economic Review, 104(2), 379-421.

Cerutti, E., Claessens, S. \& Ratnovski, L. (2014). Global liquidity and drivers of cross-border bank flows. IMF Working Paper 14/69.

Cerutti, E., Claessens, S. \& Laeven, L. (2017). The use and effectiveness of macroprudential policies: New evidence. Journal of Financial Stability, 28, 203-224.

Christensen, I., Meh, C. \& Moran, K. (2011). Bank leverage regulation and macroeconomic dynamics [manuscrito].

Claessens, S., Kose, M. A. \& Terrones, E. M. (2011). Understanding financial cycles. VoxEU.org.

Clarida, R., Galí, J. \& Gertler, M. (2000). Monetary policy rules and macroeconomic stability: Evidence and some theory. Quarterly Journal of Economics, 115(1), 147-180.

Collard, F., Dellas, H., Diba, B. \& Loisel, O. (2017). Optimal monetary and prudential policies. American Economic Journal: Macroeconomics, 9(1), 40-87.

Constâncio, V. (2017, May). The future of monetary policy frameworks. Conferencia impartida en el Instituto Superior de Economía e Gestão.

Darracq, M., Kok, C. \& Rodríguez-Palenzuela, D. (2011). Macroeconomic propagation under different regulatory regimes: Evidence from an estimated DSGE model for the Euro Area. International Journal of Central Banking, 7(4), 49-113.

Darracq, M., Kok, C. \& Rancoita, E. (2019). Macroprudential policy in a monetary union with cross-border banking. ECB Working paper Series No. 2260.

de Blas, B. (2009). Can financial frictions help explain the performance of the US Fed? The BE Journal of Macroeconomics, 9(1), artículo 27.

de Blas, B. (2020). Financial integration and the sovereign-bank loop: What role for macroprudential policy? [manuscrito].

De Paoli, B. \& Paustian, M. (2017). Coordinating monetary and macroprudential policies. Journal of Money, Credit and Banking, 49(2-3), 319-349.

Dées, S. (2016). Credit, asset prices and business cycles at the global level. ECB Working Paper Series No. 1895.

Dehmej, S. \& Gambacorta, L. (2019). Macroprudential policy in a monetary union. Comparative Economic Studies, 61(2), 195-212.

Edge, R. M. \& Liang, N. (2019). New financial stability governance structures and central banks. Finance and Economics Discussion Series (FEDS) Working Paper No. 19.

Edge, R. M. \& Liang, N. (2020). Financial stability committees and Basel III macroprudential capital buffers. Finance and Economics Discussion Series (FEDS) Working Paper No. 16.
ESRB, European Stability Risk Board (2013). Recomendación ESRB/2013/1. Boletín oficial de la Unión Europea.

Estrada, Á., Molina, L., Sánchez, P. \& Viani, F. (2017). Hacia una gestión eficiente de los flujos de capitales. Artículos analíticos del Banco de España.

Farhi, E. \& Werning, I. (2016). A theory of macroprudential policies in the presence of nominal rigidities. Econometrica, 84(5), 1645-1704.

Farhi, E. \& Werning, I. (2020). Taming a Minsky cycle [manuscrito].

Fondo Monetario Internacional (2013). The interaction of monetary and macroprudential policies.

Fondo Monetario Internacional (2015). Monetary policy and financial stability.

Freixas, X., Laeven, L. \& Peydró, J. L. (2015). Systemic risk, crises, and macroprudential regulation. The MIT Press.

FSB-IMF-BIS (2011). Macroprudential policy tools and frameworks. Update to G20 Finance Ministers and Central Bank Governors.

Galati, G. \& Moessner, R. (2013). Macroprudential policy A literature review. Journal of Economic Surveys, 27(5), 846-878.

Gebauer, S. \& Mazelis, F. (2020, May). Macroprudential regulation and leakage to the shadow banking sector. ECB Working Paper Series No. 2406.

Gertler, M. \& Gilchrist, S. (1993). The role of credit market imperfections in the monetary transmission mechanism: Arguments and evidence. The Scandinavian Journal of Economics, 95(1), 43-64.

Gertler, M. \& Gilchrist, S. (1994). Monetary policy, business cycles, and the behavior of small manufacturing firms. The Quarterly Journal of Economics, 109(2), 309-340.

Jordà, Ò., Schularick, M. \& Taylor, A. M. (2017). Macrofinancial history and the new business cycle facts. In M. Eichenbaum y J. A. Parker (eds.), Macroeconomics Annual 2016 (pp. 213-282).

Kannan, P., Rabanal, P. \& Scott, A. (2009). Monetary and macroprudential policy rules in a model with house price booms. IMF Working Paper No. 09/251.

Mencía, J. \& Saurina, J. (2016). Política macroprudencial: objetivos, instrumentos e indicadores. Documentos ocasionales de Banco de España N. ${ }^{0} 1601$.

Mendoza, E. G. (2016). Macroprudential policy: Promise and challenges. PIER Working Paper No. 16-20.

Mersch, Y. (2020). Asset price inflation and monetary policy [Discurso en la celebración del $60^{\circ}$ aniversario de INVESTAS, 27/1/20].

Nier, E. W. \& Kang, H. (2016). Monetary and macroprudential policies - exploring interactions. BIS papers No. 86, 27-38.

Portes, R. (2014). Macroprudential policy and monetary policy. In D. Schoenmaker (ed.), Macroprudentialism: A New Vox eBook. 
Quint, D. \& Rabanal, P. (2014). Monetary and macroprudential policy in an estimated DSGE model of the Euro Area. International Journal of Central Banking, 10(2), 169-236.

Rajan, R. (2005). Has financial development made the world riskier? [Jackson Hole Conference Proceeding, 313-369, Federal Reserve Bank of Kansas City].

Rey, H. (2013). Dilemma not Trilemma: the Global financial cycle and monetary policy independence [Simposio de Jackson Hole, Federal Reserve Bank of Kansas City].

Rey, H. (2014, November). The international credit channel and monetary autonomy [Mundell-Fleming Lecture, IMF Annual Research Conference].

Rubio, M. \& Carrasco-Gallego, J. A. (2015). Macroprudential and monetary policy rules: A welfare analysis. The Manchester School, 83(2), 127-152.

Rubio, M. \& Yao, F. (2019). Macroprudential policies in a low interest rate environment. Journal of Money, Credit and Banking, 52(6), 1565-1591.

Schoenmaker, D. (2014). Macroprudentialism: A new Vox eBook. VoxEU.org.

Silvo, A. (2019). The interaction of monetary and macroprudential policies. Journal of Money, Credit and Banking, 51(4), 859-894.

Smets, F. (2014). Financial stability and monetary policy: How closely interlinked? International Journal of Central Banking, 10(2), 263-300.

Stein, J. C. (2011). Monetary policy as financial-stability regulation. NBER Working Paper No. 16883.
Stock, J. H. \& Watson, M. W. (2002). Has the business cycle changed and why? NBER Macroeconomics Annual 2002, 17, 159-218.

Svensson, L. E. O. (2011). Inflation targeting. In B. M. Friedman \& M. Woodford (eds.), Handbook of Monetary Economics (pp. 1237-1302).

Svensson, L. E. O. (2014, November). Monetary policy trade-offs in CESEE [Conferencia sobre Integración económica europea (CEEI) Viena].

Svensson, L. E. O. (2018). Monetary policy and macroprudential policy: Different and separate? Canadian Journal of Economics, 51(3), 802-827.

Tayler, W. J. \& Zilberman, R. (2016). Macroprudential regulation, credit spreads and the role of monetary policy. Journal of Financial Stability, 26, 144-158.

The Economist (2020). The ECB considers counting owner-occupied housing in inflation. The Economist, consultado el 17/10/20.

Tinbergen, J. (1952). On the theory of economic policy. North-Holland Publishing Company.

Van der Ghote, A. (2018). Coordinating monetary and financial regulatory policies. ECB Working Paper Series No. 2155.

Weidmann, J. (2014, February). All for one and one for all? The roles of microprudential, macroprudential, and monetary policy in safeguarding financial stability [Discurso en el Bundesbank Symposium on Financial Stability and the Role of Central Banks]. 


\section{En el próximo número de \\ Información Comercial Española. Revista de Economia}

\section{España frente al reto industrial}

Últimos números publicados:

La Unión Europea tras la pandemia

La fiscalidad internacional ante los retos de la globalización, la digitalización y el envejecimiento

Números en preparación:

Megalópolis: desarrollo urbano y economía

Industria y transición energética

Transición digital en la industria europea

La financiación de la industria

La política de innovación, un nuevo centro de la política industrial

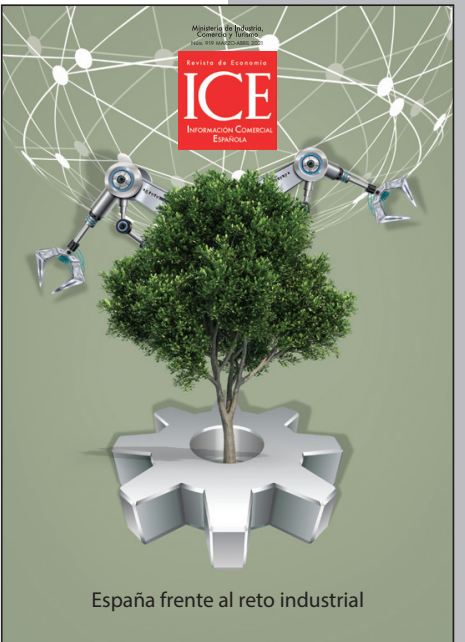

Coordinadores: Galo Gutiérrez Monzonís y Rafael Myro Sánchez 